\title{
Object Recognition based on Template Matching and Correlation Method in Hyperspectral Images
}

\author{
Divya Gupta \\ M. Tech \\ Computer Science \& Engineering \\ MIET Meerut, India
}

\author{
Sudhir Goswami \\ Asst. Professor \\ Computer Science \& Engineering \\ MIET Meerut, India
}

\begin{abstract}
In past recent years, image processing of hyperspectral images have become more popular than earlier. New methods, techniques and logics are being evolved for extracting more information from a digital image, as the existing techniques of image processing and analyzing techniques are not quenching the thirst of today's demand. The research of image processing based on traditional low resolution image has already not satisfied the need for people to get more accurate information from high resolution hyperspectral images. The today's demand is, to get more information about some particular things and modify about a particular region from a digital hyperspectral image, this is particularly central to the urban plan and disaster observation. On the basis of analysis of the conventional techniques for information extracting from a digital image, a method of extraction particular object in hyperspectral image based on feature template correlation is proposed. There are three different pars in this technique: building the template, image match and template correlations, and object recognition. The methods are applied to several high-resolution example images, and vehicles as example object in the image are extracted and recognized. Those examples illuminate that the method proposed in this paper is very effective and accurate.
\end{abstract}

\section{Keywords}

Correlation, Matching, Extraction, High resolution, Object Recognition, SD (Standard Deviation) Hybrid filters, Weiner filters, Noise, Impulse noise, photoelectric noise.

\section{INTRODUCTION}

As the improvement increased of improvement of spatial domain of digital hyper spectral images, the more information can be obtained about an object on earth. Based on conventional hyper spectral image processing techniques, different type of terrain can be classified, like farms, forests and city etc. When the resolution of hyper spectral image approaches to 1 meter or less than 1 meter, small objects can be identified on the surface clearly, such as houses, vehicles, and so on. It is not easy to differentiate those little objects from image background by conventional image processing techniques. Today, there are many studies on manmade objects (pavements, home, vehicles) recognition in the highresolution. Object identification algorithm in optical camera image processing are applied to the hyperspectral image so that the spatial resolution of image could improve, but there are numerous different problem faced to hyperspectral image processing and optical camera image processing, that are (i) the spatial resolution of hyperspectral image is relatively low although much enhanced. (ii) the hyperspectral images are acquired with different viewpoint angle, view field. (iii) the SNR of the hyperspectral image is relatively low. (iv) the object in hyperspectral image usually has scale, translation and distortion. (v) the ratio of the number of object pixel and whole image pixel is quite small.

As the object on the earth are of several types, from a big town to a tiny vehicle, and one object has different appearance on the hyperspectral image because of different view point, view field, view angle and changed weather condition. The algorithm based on supervised or unsupervised methods has good performance to classify large terrain objects. But for small objects in the remote sensing image, the algorithm for recognition should be paid more attention to study.

\subsection{Image Processing}

In the field of image processing [1], the image may be of degraded quality, the reasons behind it, are many, likedifferent weather conditions, poor quality equipment, data loss while transmission. So, for analyzinga image with high accuracy and performance we need image restoration. Image restoration plays a vital role in image processing filed. For a human being the image can be recreated by removing the noise or the irrelevant data from the original image. Any image captured by equipment is suspected to be degraded from its actual quality, it may be because of the environment in which the image was acquired or because of poor transmission. The main agenda of processing and improving the quality of an image is to restore it to its actual quality, so that it can be analyzed effectively.

\subsection{Types of Noise}

Noise in a digital image [2] is the unwanted effect produced in the image. During image capturing or transmission, there are so many factors are responsible for creating noise in the image. It depends on the type interruption, the noise can affect the image to different limit. Usually our target is to remove certain kind of noise. So we identify particulartype of noise and apply different algorithms to vanish off the noise. Noise in the image can be classified as Impulse noise (Salt-andpepper noise), Amplifier noise (Gaussian noise), Shot noise, Quantization noise (uniform noise), Film grain, on-isotropic noise, Multiplicative noise (Speckle noise) and Periodic noise. Below, we have discussed different types of noises that may be found in a hyperspectral image.

\subsubsection{Impulse Noise:}

Salt and Pepper noise is consider as the impulse noise .This type of noise is generally created by the malfunctioning of the pixel elements in the camera sensors, faulty memory locations, or timing errors in the digitization process. The images corrupted by the impulse noise the noisy pixels can take only the maximum and the minimum values in the dynamic range.Salt and pepper noise will have the dark pixel value in dark region and the dark region will have the dark pixel. 


\subsubsection{Gaussian Noise}

The quantum fluctuations are generally caused by the lighter part of the image through the dominant noise. There are the variations in number of photons; this type of noise is called photon shot noise. Gaussian noise has a root-mean-square value proportional to the square root of the image intensity. The noises having different pixels are independent of each other.

\subsubsection{Electronic Noise}

This type of noise is generally created by the electronic motion of the component of the imaging system.

\subsubsection{Photoelectric Noise:}

This type of noise is generally created by the statically nature of light and photo electronic conversion.

\subsubsection{Film grain noise}

It is due to randomness of silver halide grains in the film used for recording.

\subsubsection{Thermal noise}

The thermal energy is a stochastic source of CCD in electronic component. Due to thermal vibration electrons can be freed from CCD material itself .Due to cooling down the CCD chip the thermal noise is decrease in the original noise. As the number of thermal electrons increases, the integration time also increases. The probability distribution of thermal electrons is also a Poisson process where the rate parameter is increasing function of temperature. The dark current average, it does not reduce the dark current standard deviation.

\subsection{Image Restoration}

Images are produced to record or display useful information. Due to imperfections in the imaging and capturing process, however, the recorded image invariably represents a degraded version of the original scene. The undoing of these imperfections is crucial to many of the subsequent image processing tasks. There exists a wide range of different degradations that need to be taken into account, covering for instance noise, geometrical degradations (pin cushion distortion), illumination and color imperfections (under/overexposure, saturation), and blur.

\subsubsection{Application of Image Restoration}

In the field of image processing [3][4], the image may be of degraded quality, the reasons behind it, are many, likedifferent weather conditions, poor quality equipment, data loss while transmission. So, for analyzing an image with high accuracy and performance we need image restoration. . Image restoration plays a vital role in image processing field. For a human being the image can be recreated by removing the noise or the irrelevant data from the original image. Any image captured by an equipment is suspected to be degraded from its actual quality, it may be because of the environment in which the image was acquired or because of poor transmission. The main agenda of processing and improving the quality of an image is to restore it to its actual quality, so that it can be analyzed effectively.

(i) The application area of image restoration is widely used in every aspect of image processing. The image restoration is used at a large scale in analyzing hyperspectral images, by different space agencies like ISRO, NASA.

(ii) The images acquired by the cameras installed in the equipments or in satellites captures good images but the surrounding conditions put some noise in the digital image, the reason may be bad environment or the week transmission techniques. Furthermore the astronomical digital imaging degradation difficulty is usually characterized by Poisson noise, Gaussian noise etc.

(iii) Another important application of restoration technique is to restore aging and deteriorated films. The thought of motion picture reestablishment is often associated with digital techniques used not only to reduce scratches and dust from old movies, but also to colorize black and white films.

(iv) Image processing is also used in medical field whether it is a surgery or analysing bone density. Restoration has been used for filtering of Poisson distributed film-grain noise in chest $\mathrm{X}$-rays, mammograms and angiographic images, and to remove additive noise in Magnetic resonance Imaging.

(v) The expanding area of application for image restoration in image processing is that in the area of image and video coding. As techniques are developed to improve coding effectiveness, and decrease the bit rates of coded images. Much has been completed to model these kinds of artifacts and grow ways of restoring coded digital images as a post-processing step to be performed after decompression.

\subsection{Filter}

Mostly images are degraded to some limit by noise, that is unexplained variation in data: noises in a digital image intensity which are either not interpretable or not of interest. Usually image analysis is simplified if this noise can be filtered out. In an analogous way filters are used in chemistry to liberate liquids from suspended impurities by passing them through a layer of sand or charcoal. People working in signal processing have extended the meaning of the term filter to include operations which accentuate features of interest in data. Employing this broader definition, digital image filters may be used to highlighting edges - that is, boundaries between objects or parts of objects in images. Filters provide a help to visual interpretation of images, and can also be used as a precursor to further digital processing, such as segmentation.

\subsubsection{Types of Filters}

De-noising [5] a digital image is very beneficial and very much required task in digital image processing for the analyzing a digital images. There are different types image denoising methods are available, but the best one should vanish the noise offfully from the input image, while preserving the properties of image. De-noising techniques can be linear as well as non-linear. Where linear methods are fast enough, but they do not keep the details of the images, whereas the nonlinear techniques preserve the properties of the images. In a big picture, De-noising filters can be divided in the below categories.

- Averaging filter

- Order Statistics filter

- Adaptive filter

\section{Mean filter-}

Mean filter is an Averaging Linear Filter. Here the filter calculates the average value of the distorted 
image in a predecided area. After this the center pixel intensity value is replaced by that average value. This process is repeated for all pixel values in the image.

\section{Median Filter-}

Median filter is a best Order Statics non- linear Filter, whose response is based on the ranking of pixel values enclosed in the filter region. Median filter is quite admired for decreasing certain types of noise. Here the center value of the pixel is replaced by the median of the pixel values under the filter region. Median filter is good for salt and pepper noise. These filters are widely used as smoothers for image processing, as well as in signal processing. A major benefit of the median filter over linear filters is that the median filter can remove the effect of input noise values with extremely massive magnitudes.

\section{Order Statistics Filter-}

Order-Statistics filters are non-linear filters whose response depends on the ordering of pixels encompassed by the filter area. When the center value of the pixel in the image area is replaced by 100th percentile, the filter is called Max-filter. On the other hand, if the same pixel value is replaced by 0 th percentile, the filter is termed as Minimum Filter.

\section{Adaptive Filter}

Adaptive Filterscan change their behavior on the basis of statistical characteristics of the image area, encompassed by the filter region.BM3D is an adaptive filter. It is a nonlocal image modeling technique based on adaptive, high order group-wise models.

\section{Homomorphic Filter}

A homomorphic filtering technique [6] significantly improves target recognition performance on images with difficult illumination conditions.Homomorphic Filter approach for image processing is very well known as a way for image dynamic range and increasing contrast. According to this approach, input is assumed to have2 multiplicative elements: background and details. The standard problem in processing such signals involves logarithm operation, division on 2 elements by implementing low frequency and high-pass filters, addition of evaluations multiplied by different gain coefficients, and exponent calculation.

The illumination component of an image is generally characterized by slow spatial variation while the reflectance component of an image tends to vary abruptly.

These characteristics lead to associating the low frequencies of the Fourier transform of the natural log of an image with illumination and high frequencies with reflectance.

Even though these assumptions are approximation at best, a good deal of control can be gained over the illumination and reflectance components with a homomorphic filter.

Homomorphic filtering is a method in which the illumination and reflectance components can be filtered individually.

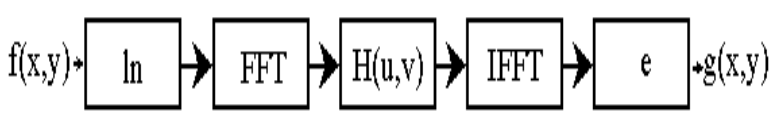

\subsection{Correlation matching}

Correlation [7] is a basic operations that is used extract information from images. In some sense it is the simplest operation that we can perform on an image, but it is extremely useful. Moreover, because it is simple, so it can be analyzed and understood very well, and itis also easy to implement and can be computed very efficiently. Correlation has two key features: shift-invariant, and they are linear. Shift-invariant means that we perform the same operation at every point in the image. Linear means that this operation is linear, that is, we replace every pixel with a linear combination of its neighbors. These two properties make these operations very simple.

A Mathematical Definition for Correlation It's helpful to write this all down more formally. Suppose F is a correlation filter. It will be convenient notationally to suppose that $\mathrm{F}$ has an odd number of elements, so we can suppose that as it shifts, its center is right on top of an element of I. So we say that F has $2 \mathrm{~N}+1$ elements, and that these are indexed from $-\mathrm{N}$ to $\mathrm{N}$, so that the center element of $F$ is $F(0)$. Then we can write:

$$
F \circ I(x)=\sum_{i=-N}^{N} F(i) I(x+i)
$$

where the circle denotes correlation. With this notation, we can define a simple box filter as:

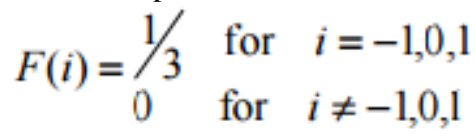

\subsubsection{Matching with Correlation}

Part of the power of correlation is that we can use it, and related methods, to find locations in an image that are similar to a template. To do this, think of the filter as a template; we are sliding it around the image looking for a location where the template overlaps the image so that values in the template are aligned with similar values in the image. First, we need to decide how to measure the similarity between the template and the region of the image with which it is aligned. A simple and natural way to do this is to measure the sum of the square of the differences between values in the template and in the image. This increases as the difference between the two increases. For the difference between the filter and the portion of the image centered at $\mathrm{x}$, we can write this as:

$$
\begin{aligned}
\sum_{i=-N}^{N}(F(i)-I(x+i))^{2} & =\sum_{i=-N}^{N}\left(F^{2}(i)+I^{2}(x+i)-2 F(i) I(x+i)\right) \\
& =\sum_{i=-N}^{N}\left(F^{2}(i)\right)+\sum_{i=-N}^{N}\left(I^{2}(x+i)\right)-2 \sum_{i=-N}^{N}(F(i) I(x+i))
\end{aligned}
$$

As shown, we can break the Euclidean distance into three parts. The first part depends only on the filter. This will be the same for every pixel in the image. The second part is the sum of squares of pixel values that overlap the filter. And the third 
part is twice the negative value of the correlation between $\mathrm{F}$ and I. We can see that, all things being equal, as the correlation between the filter and the image increases, the Euclidean distance between them decreases. This provides an intuition for using correlation to $149162536 \ldots 11 / 2468$ $1012 \ldots$ match a template with an image. Places where the correlation between the two is high tend to be locations where the filter and image match well. This also shows one of the weaknesses of using correlation to compare a template and image. Correlation can also be high in locations where the image intensity is high, even if it doesn't match the template well.

\subsubsection{TwoD Correlation}

Images are 2D [8], so we really want to perform correlation in 2D. The basic idea is the same, except that the image and filter are now 2D. We can suppose that our filter is square and has an odd number of elements, so it is represented by a $(2 \mathrm{~N}+1) \mathrm{x}(2 \mathrm{~N}+1)$ matrix. We don't lose anything with these assumptions, because we can take any filter and pad it with zeros to make it square with an odd width. Padding a filter with zeros does not change its behavior. Given a square filter, we can compute the results of correlation by aligning the center of the filter with a pixel. Then we multiply all overlapping values together, and add up the result. We can write this as:

$$
F \circ I(x, y)=\sum_{j=-N}^{N} \sum_{i=-N}^{N} F(i, j) I(x+i, y+j)
$$

\section{REVIEW OF LITERATURE}

In this paper [9], the writers has fused several detection algorithms together, the target detection in hyperspectral images is important in many applications including search and rescue operations, defence systems, mineral exploration and border security. For this purpose, several target detection algorithms have been proposed over the years, however, it is not clear which of these algorithms perform best on real data and on sub-pixel targets, and moreover, which of these algorithms have complementary information and should be fused together. The goal of this study is to detect the nine arbitrarily placed sub-pixel targets, from seven different materials from a $1.4 \mathrm{~km}$ altitude. For this purpose, eight signature-based hyperspectral target detection algorithms, namely the GLRT, ACE, SACE, CEM, MF, AMSD, OSP and HUD, and three anomaly detectors, namely RX, Maxmin and Diffident, were tested and compared. Among the signaturebased target detectors, the three best performing algorithms that have complementary information were identified. Finally these algorithms were fused together using four different fusion algorithms. Our results indicate that with a proper fusion strategy, five of the nine targets could be found with no false alarms.

In this paper [10],the main target of is to demonstrate that detectors behave differently for different images and targets and to propose a novel approach to proper detector selection. To choose the algorithm, we analyze image statistics, the target signature, and the target's physical size, but we do not need any type of ground truth. We demonstrate our ability to evaluate detectors and find the best settings for their free parameters by comparing our results using the following stochastic algorithms for target detection: the constrained energy minimization (CEM), generalized likelihood ratio test (GLRT), and adaptive coherence estimator (ACE) algorithms. We test our concepts by using the dataset and scoring methodology of the Rochester Institute of Technology (RIT)
Target Detection Blind Test project. The results show that our concept correctly ranks algorithms for the particular images and targets including in the RIT dataset.

In this paper [11], various dimensions of detection in Hyperspectral Imaging are discussed along with its vast application area. Hyperspectral Imaging is a recent trend in the field of remote sensing. Over the past few years hyperspectral imaging has been emerged as rapidly developing technology to process and analyze the images. This technique has gained interest in wide area of applications such as food processing, mineralogy, environment, vegetation, astronomy, surveillance, eye care, chemical imaging, military and so on.. This article presents the overview of hyperspectral imaging, outlines the energy distribution from source to sensor, discussing about the radiation interaction with the earth, summarizing the differences between multispectral and hyperspectral data and finally briefing the hyperspectral imaging algorithms in target detection.

In this paper [12] - In this paper a problem is, solution is achieved for clouds removal for better analysis of satellite images. In recent years, on average about $35 \%$ of cloud covers are generally present in optical satellite images. To get cloudfree satellite images for analyses of current land cover and land-cover change cloud deletion method based on information cloning is introduced. The method removes cloud-contaminated portions of a satellite image and then clones information from cloud-free patches to their corresponding cloud-contaminated patches under the assumption that land covers change insignificantly over a short period of time. To identify exact location of cloud contaminated region, cloud detection based on window based thresholding technique is introduced. The proposed information cloning algorithm is used to recreate the missing data after removing the cloud-contaminated area. By replacing cloud contaminated target image with cloud- and shadow-free parts from the reference image, the information reconstruction is performed. This approach results in cloud removed images and is tested for various input images.

\section{PROPOSED METHODOLOGY}

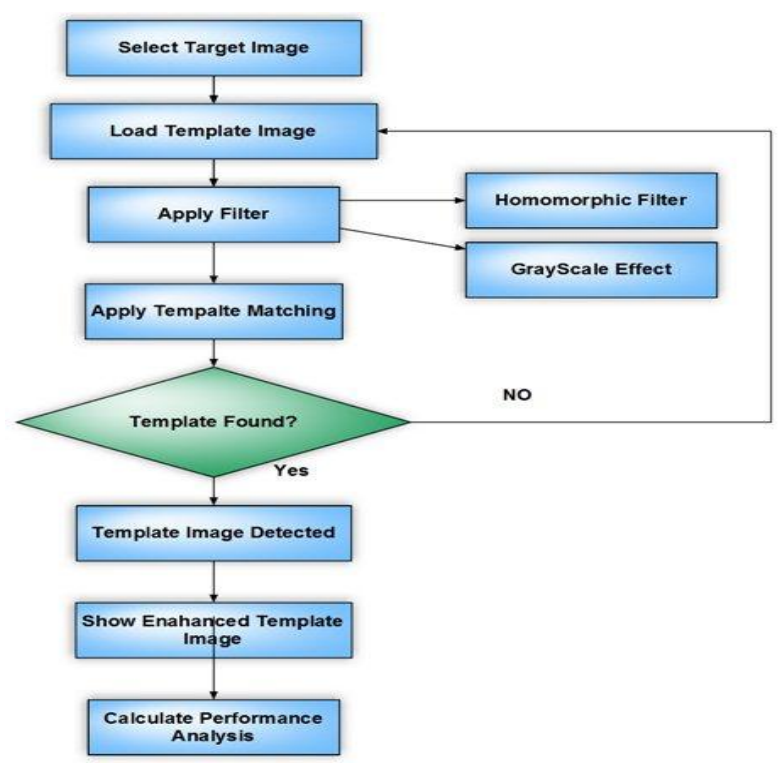

Figure 1: Flowchart of Research Methodology 
First of all the original image is read and converted to grayscale, so that the filters can be applied on it. Now a homomorphic filtering technique is applied on to improve the target recognition performance significantly, even with difficult illumination condition. Filtering the intensities even in frequency domains enhances the quality of an image to a great extent. Once the target is loaded, being small has damaged intensities due to which larger picture of the template is degraded. To overcome this problem we have to apply some technique, so a Median filter is applied so that we can have a better target template. Each output pixel contains the median value in the $\mathrm{m} \times \mathrm{n}$ neighborhood around the corresponding pixel in the input image. Median filtering is a nonlinear operation which is often used in image processing to reduce "salt and pepper" noise. A median filter is more effective than convolution when the goal is to simultaneously reduce the noise and preserve edges. But in the image we still have some kind of noise that may affect our task or may lead to a poor performance result. So to remove the Gaussian noise that is present in the image, we apply another filter, Gaussian Low Pass Filter. This filter is then applied over the whole image with replicate boundary options. (Input array values outside the bounds of the array are assumed to equal the nearest array border value). Now the Correlation is found between the 2 by applying the two-dimensional convolution of matrices or images normally as well as after flipping it. Depending on the above data a score is calculated using its convolution values and SD (Standard Deviation). The intensity or the position in the image at which the score was maximum, is considered as the target point. If the calculated score there, is greater than the threshold, the bounding box is made indicating the position at which the template is supposed to be found. Then the precision, recall, similarity, F1 \& accuracy is calculated for the input image and the template.

\section{The steps of proposed work}

1) The original image is read and converted to gray scale.

2) A homomorphic filtering technique is applied and thus significantly improve target recognition performance on images with difficult illumination conditions.Filtering the intensities even in frequency domains enhances the quality of an image to a great extent.

3) We can crop a template from the same image or load it from somewhere else.

4) Once the target is loaded,being small has damaged intensities due to which larger picture of the template is degraded.

5) Now a median filter is applied.Each output pixel contains the median value in the $\mathrm{m} \mathrm{x} \mathrm{n}$, neighborhood around the corresponding pixel in the input image.

6) We define a special filter named Gaussian low pass filter to reduce or remove Gaussian noise present in the image.

7) This filter is then applied over the whole image with replicate boundary options. (Input array values outside the bounds of the array are assumed to equal the nearest array border value.)

8) Correlation is then found between the 2 by applying the $2 \mathrm{D}$ convolution of matrices or images normally as well as after flipping it

9) Depending on the above data a score is calculated using its convolution values and standard deviation.

10) The intensity or the position in the image at which the score is maximum, is considered to be the point. If the score there is greater than the threshold the bounding box is made indicating the position at which the template is supposed to be found.
11)Then the precision,recall,similarity, F1 \& accuracy are calculated for the input image and the template.

\section{EXPERIMENTAL RESULTS}
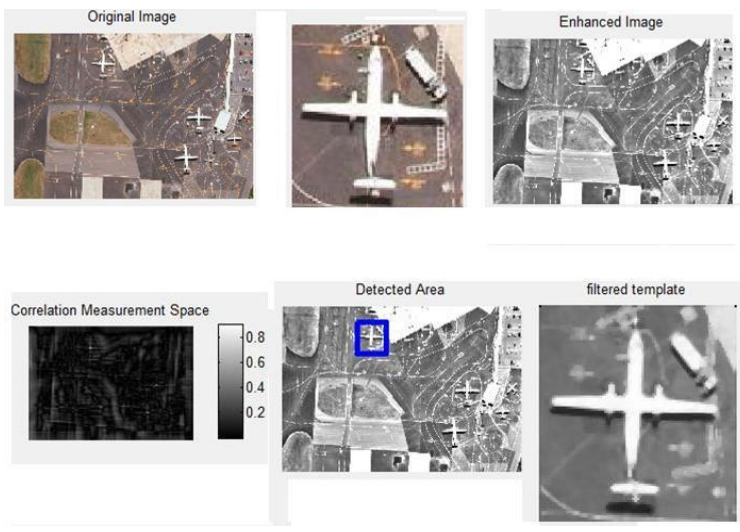

Figure 2: Original image, area to be detected, enhanced image, Correlation measurement, area detected, filtered template (from top to bottom and left to right)

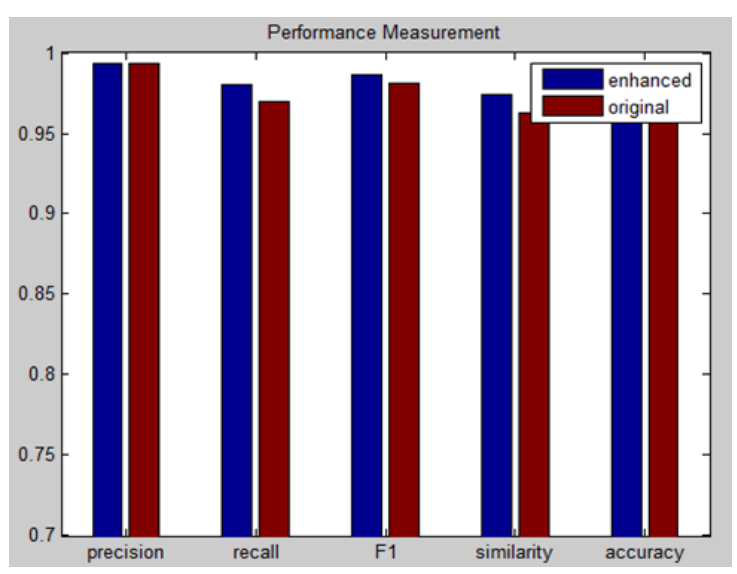

Figure 3: Performance Measurement (for aero plane ex.)

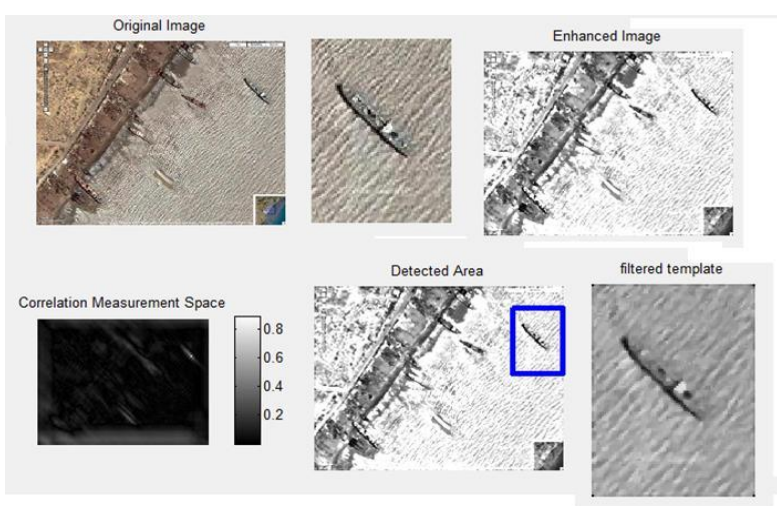

Figure 4: Original image, area to be detected, Enhanced image, Correlation measurement, area detected, filtered template (from top to bottom and left to right) 


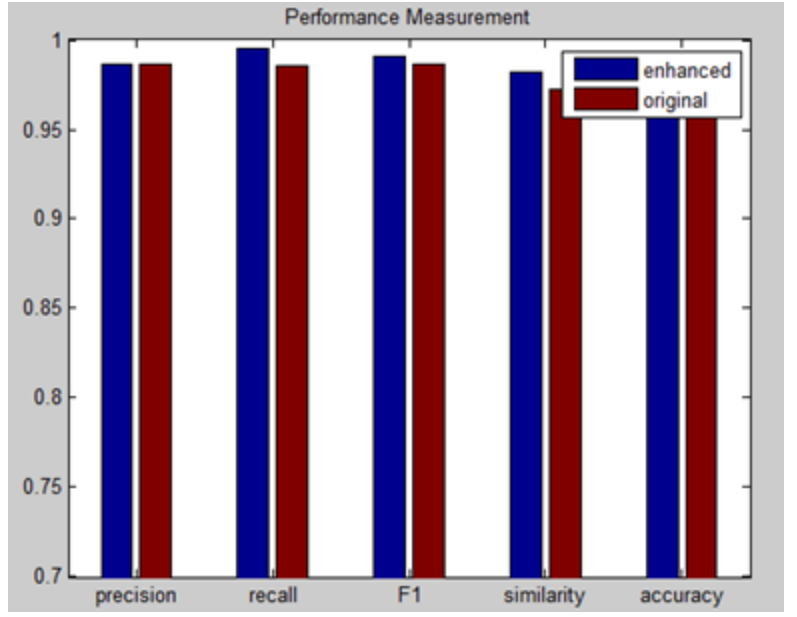

Figure 5 :Performance Measurement (for ship ex.)

\subsection{Result}

After analyzing performance measurement graph, it can be said that the techniques we have used here are advanced and accurate enough that it can detect any hyperspectral image whether it is distorted or not.

\section{CONCLUSION}

In this paper, it is evident that we have detected our target template with exact match and with good accuracy after applying image restoration and correlation technique, although it is not easy to detect something a particular target when we are dealing with hyperspectral images. But with the techniques we have for image processing are capable enough to deal with such problems. And as a future work, more accuracy can be achieved with minimum execution time.

\section{REFERENCES}

[1] SenihaEsen, AhmetKarakaya; "Fusion of target Detection Algorithms In Hyperspectral Images" International Journal of Intelligent System and Applications In Engineering, Vol 4, No 4 (2016)

[2] DimitrisManolakis, Eric Truslow, Michael Pieper, Thomas Cooley, Michael Brueggeman, "Detection Algorithms in Hyperspectral Imaging Systems: An Overview of Practical Algorithms," IEEE Signal Processing Magazine, vol. 31, no. 1, 2014.

[3] Yuval Cohen, Yitzhak August, Dan G. Blumberg, Stanley R. Rotman"Evaluating Subpixel Target
Detection Algorithms in Hyperspectral Imagery", Journal of Electrical and Computer Engineering Volume 2012 (2012),

[4] Tan, J.L. Meat quality evaluation by computer vision. J. Food Eng. 2004, 61, 27-35. 4. O'sullivan, M.G.; Byrne, D.V.; Martens, H.; Gidskehaug, L.H.; Andersen, H.J.; Martens, M. Evaluation of pork colour: Prediction of visual sensory quality of meat from instrumental and computer vision methods of colour analysis. Meat Sci. 2003, 65, 909-918.

[5] Faucitano, L.; Huff, P.; Teuscher, F.; Gariepy, C.; Wegner, J. Application of computer image analysis to measure pork marbling characteristics. Meat Sci. 2005, $69,537-543$.

[6] Rodbotten, R.; Nilsen, B.N.; Hildrum, K.I. Prediction of beef quality attributes from early post mortem near infrared reflectance spectra. Food Chem. 2000, 69, 427436.

[7] B. Datt, T.R. McVicar, T.G. Van Niel, D.L.B. Jupp, J.S Pearlman, "Preprocessing EO-1 Hyperion hyperspectral data to support the application of agricultural indexes", Geoscience and Remote Sensing, IEEE Transactions, vol. 41, no. 6, pp. 1246 - 1259, 2003.

[8] Dr. Vijayalakshmi M.N , M. Senthilvadivu,"An Assessment of Hyperspectral Imaging and Target detection",International Journal of Advanced Research in Computer and Communication Engineering Vol. 3, Issue 8, August 2014

[9] h. Akbari, et al., "Hyperspectral Image Segmentation and its Application in Abdominal Surgery," International Journal of Functional Informatics and Personalised Medicine, vol. 2, pp. 201-216, 2009.

[10] A.A. Gowena,, C.P. O'Donnella , P.J. Cullenb , G. Downeyc and J.M. Friasb;"Hyperspectral imaging e an emerging process analytical tool for food quality and safety control, "Elsevier, Trends in Food Science \& Technology 18 (2007) 590e598

[11] Saranya M; "Cloud Removal from Satellite Images Using Information Cloning", Saranya M, International Journal of Computer Science and Mobile Computing, Vol.3 Issue.2, February- 2014, pg. 681-688

[12] A.Majumder and S. Irani, "Perception-based contrast enhancement of images," ACM Trans. Appl. Percpt., 4(3): (2007) 